\title{
Direct Binding of the Corrector VX-809 to Human CFTR NBD1: Evidence of an Allosteric Coupling between the Binding Site and the NBD1:CL4 Interface ${ }^{\text {s }}$
}

\author{
Rhea P. Hudson, Jennifer E. Dawson, P. Andrew Chong, Zhengrong Yang, Linda Millen, \\ Philip J. Thomas, Christie G. Brouillette, and Julie D. Forman-Kay
}

Molecular Medicine, Hospital for Sick Children, Toronto, Ontario, Canada (R.P.H, J.E.D., P.A.C., J.D.F.-K.); Department of Biochemistry, University of Toronto, Toronto, Ontario, Canada (J.D.F.-K.); Center for Structural Biology (Z.Y., C.G.B.) and Department of Chemistry (C.G.B.), University of Alabama at Birmingham, Birmingham, Alabama; and Department of Physiology, UT Southwestern Medical Center, Dallas, Texas (L.M., P.J.T.).

Received January 17, 2017; accepted May 17, 2017

\section{ABSTRACT}

Understanding the mechanism of action of modulator compounds for the cystic fibrosis transmembrane conductance regulator (CFTR) is key for the optimization of therapeutics as well as obtaining insights into the molecular mechanisms of CFTR function. We demonstrate the direct binding of VX-809 to the first nucleotide-binding domain (NBD1) of human CFTR. Disruption of the interaction between $\mathrm{C}$-terminal helices and the NBD1 core upon VX-809 binding is observed from chemical shift changes in the NMR spectra of residues in the helices and on the surface of $\beta$-strands S3, S9, and S10. Binding to VX-809 leads to a significant negative shift in NBD1 thermal melting temperature $\left(T_{m}\right)$, pointing to direct VX-809 interaction shifting the NBD1 conformational equilibrium. An inter-residue correlation analysis of the chemical shift changes provides evidence of allosteric coupling between the direct binding site and the NBD1:CL4 interface, thus enabling effects on the interface in the absence of direct binding in that location. These NMR binding data and the negative $T_{m}$ shifts are very similar to those previously reported by us for binding of the dual corrector-potentiator CFFT-001 to NBD1 (Hudson et al., 2012), suggesting that the two compounds may share some aspects of their mechanisms of action. Although previous studies have shown an important role for $\mathrm{VX}-809$ in modulating the conformation of the first membrane spanning domain (Aleksandrov et al., 2012; Ren et al., 2013), this additional mode of VX-809 binding provides insight into conformational dynamics and allostery within CFTR.

\section{Introduction}

Cystic fibrosis is caused by mutations in the CFTR chloride channel (Riordan et al., 1989). The most common and severe cystic fibrosis-causing mutation in CFTR is the deletion of F508 (F508del), which is found in $90 \%$ of patients (Bobadilla et al., 2002). The loss of CFTR function in patients with F508del is due to the mutation exacerbating the already inefficient folding and processing of CFTR, targeting it for degradation. The few F508del-CFTR molecules that do escape degradation show a decreased open probability (Dalemans et al., 1991).

Therapeutic efforts have targeted small molecules that counter either the processing defect (correctors) or the gating defect (potentiators). VX-809, a corrector, partially restores trafficking in F508del CFTR, increasing its plasma membrane

This work was funded by Cystic Fibrosis Foundation Therapeutics [Grants FORMAN13XX0, BROUIL08XX0, and BROUIL13XX0]; and Cystic Fibrosis Canada [Grant 3175].

https://doi.org/10.1124/mol.117.108373.

S This article has supplemental material available at molpharm. aspetjournals.org. concentration in human bronchial epithelial cells isolated from F508del homozygous patients (Van Goor et al., 2011). Although VX-809 alone does not significantly improve lung function (Clancy et al., 2012), in combination with the potentiator, VX-770, it yields a significant improvement in forced expiratory volume in 1 second values of F508del CFTR homozygous patients (Boyle et al., 2014).

Similar to other ABC transporters, CFTR contains two MSDs and two NBDs (Supplemental Fig. 1A). A large intrinsically disordered regulatory $(\mathrm{R})$ region (not shown), unique to CFTR, connects NBD1 to MSD2. Homology models (Corradi et al., 2015; Mornon et al., 2015) and recent cryo-electron microscopy structures (Zhang and Chen, 2016; Liu et al., 2017) show intracellular domains that are formed by cytosolic extensions of the transmembrane helices and are connected by shorter coupling helices referred to as intracellular loops (CLs). F508 in NBD1 makes contact with CL4, thus participating in bridging NBD1 and MSD1.

CFTR functions as an ion channel regulated by cAMPdependent protein kinase A phosphorylation of the $\mathrm{R}$ region and the regulatory insertion (RI) (Ostedgaard et al., 2001) and

ABBREVIATIONS: 1D, one dimensional; BIA, 5-bromoindole-3-acetic acid; CFTR, cystic fibrosis transmembrane conductance regulator; CL, intracellular loop; DLS, dynamic light scattering; DMSO, dimethylsulfoxide; DSC, differential scanning calorimetry; F508del, deletion of F508; HSQC, heteronuclear single quantum coherence; ID, identification; MSD, membrane spanning domain; NBD, nucleotide binding domain; PDB, Protein Data Bank; $P_{\mathrm{F}}$, Fisher test $P$ value; $\mathrm{R}$, regulatory; RE, regulatory extension; RI, regulatory insertion; $\mathrm{T}_{\mathrm{m}}$, melting temperature; WT, wild type. 
ATP binding and hydrolysis at the NBD1:NBD2 heterodimer interface (Chappe et al., 2005; Mense et al., 2006). It has been proposed that ATP- and dimerization-induced conformational changes are relayed to the MSDs via the intracellular domains, leading to pore opening and closing (Mense et al., 2006; Hwang and Sheppard, 2009; Mornon et al., 2009)

At the molecular level, F508del leads to reduced thermostability of isolated NBD1 (Protasevich et al., 2010; Wang et al., 2010) and a disruption of the NBD1:CL4 interface. It has also been suggested that F508del may impair the ability of the NBDs to dimerize, thereby destabilizing the channel (Chong et al., 2015).

The mechanism of action of VX-809 remains largely unknown. Given that VX-809 does not stabilize isolated NBD1 (Loo et al., 2013), it has been suggested to act by correcting domain-domain interface defects, in particular NBD1:CL4. Mutational correction of the NBD1:CL4 interface enhances CFTR processing (Loo et al., 2010; Thibodeau et al., 2010; Mendoza et al., 2012; Rabeh et al., 2012; He et al., 2013). Simultaneous mutational correction of NBD1 thermostability and the NBD1:CL4 interface synergistically improves CFTR processing and F508del expression levels to $50-80 \%$ of wildtype (WT) levels (Mendoza et al., 2012; Rabeh et al., 2012), implying that NBD1 thermostability and NBD1:CL4 interface defects together account for much of the defective processing in F508del. Interestingly, recent work has demonstrated that NBD1 stabilization alone is sufficient for correction and that the dual-target approach can lead to a locked open constitutively active CFTR channel (He et al., 2015).

Although docking and mutagenesis suggest that VX-809 directly restores defective NBD1:CL4 interactions (Serohijos et al., 2008; He et al., 2013; Okiyoneda et al., 2013), it also corrects multiple CL4 mutants and mutations in other CLs (He et al., 2013) and successfully restores high levels of CFTR processing to MSD1-specific mutants (Ren et al., 2013). It is unlikely that VX-809 binds at all of these conformationally disturbed sites. The ability of VX-809 to correct a diverse variety of CFTR mutations may hinge on the allosteric linkage of various regions of CFTR, such that drug binding at one site can have a stabilizing effect elsewhere.

Given the lack of clarity on the mechanisms of action of current CFTR modulators, we have used NMR spectroscopy, differential scanning calorimetry (DSC), and statistical analysis to probe the binding of VX-809 to isolated domains of human CFTR. The addition of VX-809 to WT and F508del NBD1 results in conformational changes within NBD1 that were nearly identical to those previously described for another compound, CFFT-001 (Hudson et al., 2012). DSC data show a similar reduction in melting temperature $\left(\mathrm{T}_{\mathrm{m}}\right)$ upon addition of VX-809. Taken together, these results strongly suggest that VX-809 may share some aspect of its mechanism of action with CFFT-001. We further show statistically significant changes in the CL4 cleft of NBD1 upon VX-809 binding, providing evidence for an allosteric linkage between the two regions.

\section{Materials and Methods}

Protein Expression and Purification (NMR and DSC Studies). Constructs for human NBD1 lacking the RI and regulatory extension (RE) [NBD1 $\triangle \mathrm{RI} \triangle \mathrm{RE}, 387-646, \triangle 405-436$ ), with or without F508 or H620D; and NBD2 (1193-1445, Q1280E/H1402A/L1436D/Q1411$\mathrm{D} / \mathrm{Y} 1307 \mathrm{~N} / \mathrm{S} 1255 \mathrm{~L} / \mathrm{S} 1359 \mathrm{~A})]$ were expressed as $\mathrm{His}_{6}$-small ubiquitinlike modifier fusions, cleaved with Ulp protease and further purified as described previously (Hudson et al., 2012; Vernon et al., 2017). Purified NBD constructs were stored at $4^{\circ} \mathrm{C}$ in buffer containing $12.5 \%$ glycerol before being exchanged into $50 \mathrm{mM} \mathrm{NaPhos}, \mathrm{pH} 7.25,150 \mathrm{mM} \mathrm{NaCl}$, $5 \mathrm{mM}$ ATP/ $/ \mathrm{MgCl}_{2}, 2 \%$ (v/v) glycerol, $2 \mathrm{mM}$ dithiothreitol, $10 \% \mathrm{D}_{2} 0$ with $0.05 \%$ sodium azide, and $0.5 \%$ benzamidine for NMR or into $150 \mathrm{mM}$ $\mathrm{NaCl}, 20 \mathrm{mM}$ HEPES pH 7.5, 10\% glycerol, 10\% ethylene glycol, $1 \mathrm{mM}$ Tris(2-carboxymethyl)phosphine, $2 \mathrm{mM}$ ATP, and $3 \mathrm{mM} \mathrm{MgCl}_{2}$ for DSC.

Compounds. The initial supply of VX-809 and VRT-528 (Supplemental Fig. 2) (Van Goor et al., 2006) were provided by Vertex Pharmaceuticals, Inc. (San Diego, CA). Further stocks of VX-809 were purchased from Selleckchem.com (cat. no. S1565). 5-Bromoindole-3acetic acid (BIA) was purchased from Sigma-Aldrich (St. Louis, MO) (cat. no. B68720). CFFT-001, originally synthesized by Epix Pharmaceuticals (Lexington, MA), was provided by Cystic Fibrosis Foundation Therapeutics (Bethesda, MD).

NMR Spectroscopy. NBD1 and NBD2 NMR heteronuclear single quantum coherence (HSQC) experiments were carried out at $20^{\circ} \mathrm{C}$ on a $500 \mathrm{MHz}$ Varian spectrometer (Agilent Technologies, Santa Clara, CA) equipped with a triple-resonance room-temperature probe with pulsed field gradients. Data were processed and analyzed using nmrDraw (Delaglio et al., 1995), nmrView (Johnson and Blevins, 1994), and Sparky (T.D. Goddard, D.G. Kneller; http://www.cgl.ucsf. edu/home/sparky/). The one dimensional (1D)-fluorine experiments were recorded on a $600 \mathrm{MHz}$ Varian NMR spectrometer (Agilent Technologies) on $1.5 \mathrm{mM}$ VX-809 samples with and without $500 \mu \mathrm{M}$ CFTR NBD1 WT. We used a MATLAB script to fit chemical shift perturbation-based $K_{\mathrm{d}}$ values for the binding of VX-809 to WT and F508del NBD1. Individual fits were first determined by fitting the individual curves for each residue. This was followed by global fitting during which global residuals were obtained by summing residuals of each titration pattern at a given $K_{\mathrm{d}}$ value. To avoid giving poorly fitting titration patterns more weight than well-fitting patterns, the summing of residuals of all curves are weighted by the inverse of the fitting residual.

Docking. VX-809 was computationally docked onto the NBD1 structure using the coordinates of the $2 \mathrm{PZE}$ crystal structure truncated at residue 630 to expose the proposed VX-809 binding pocket and eliminate steric clashes with $\mathrm{H} 8$ and H9. VX-809 coordinates were first generated using Open Babel (O’Boyle et al., 2011). After this, VX-809 was docked onto NBD1 using Auto Dock Vina (Trott and Olson, 2010) with a grid centered on the proposed $\beta$-strand binding site with rotatable bonds where appropriate for VX-809. Side chains in the binding pocket were also allowed flexibility around rotatable bonds. The docked compounds were viewed, and figures were generated using Pymol (Schrodinger, 2010).

DSC. DSC was performed on a VP Capillary DSC System (MicroCal; Malvern Instruments Inc., Northampton, MA) as previously described (Hudson et al., 2012). Briefly, the protein sample was exchanged into the DSC buffer (see Protein Expression and Purification) by diluting into the buffer and concentrating in an Amicon Ultra15 (10K) centrifugal filtration device (EMD Millipore, Billerica, MA) three times. VX-809 stock solution at appropriate concentrations in $100 \%$ dimethylsulfoxide (DMSO) was diluted 50 -fold into the protein sample and incubated at $4^{\circ} \mathrm{C}$ for more than 30 minutes before the DSC was performed. Data analysis was carried out using the instrument provided software.

Dynamic Light Scattering. Dynamic light scattering (DLS) was conducted using a DynaPro Dynamic Light Scattering system (Wyatt Technologies Corp., Santa Barbara, CA). The experiments were carried out at $25^{\circ} \mathrm{C}$ at a wavelength of $831.6 \mathrm{~nm}$. VX-809 stock solution in $100 \%$ DMSO was diluted 50-fold into the NMR buffer (see Protein Expression and Purification) to create a $2 \%$ DMSO solution at the appropriate compound concentration. The samples were incubated at $22^{\circ} \mathrm{C}$ for 1 hour before the DLS measurement. The data were analyzed using the instrument-provided DYNAMICS version 6 software (Wyatt Technologies Corp., Santa Barbara, CA).

CFTR Maturation. The mutation H620D was introduced into a plasmid pCMV-CFTR (a gift from J. Rommens, Hospital for Sick 
Children, Toronto, Ontario, Canada) using the QuikChange SiteDirected Mutagenesis Kit (Agilent Technologies). HEK293 cells (American Type Culture Collection, Manassas, VA) were transiently transfected using Continuum (Gemini Bio-Products, West Sacramento, CA) and grown for 24 hours at $37^{\circ} \mathrm{C}$. The transfected cells were dosed with VX-809, shifted to $30^{\circ} \mathrm{C}$, and grown for an additional 24 hours. Cells were lysed in radioimmunoprecipitation assay buffer, and the soluble fraction was analyzed by SDS-PAGE. The maturation of CFTR was visualized by Western blotting utilizing monoclonal antibody A596 (J. Riordan, University of North Carolina, Chapel Hill, NC) and quantified by the LI-COR (Lincoln, NE) Odyssey CLx Imaging System.

Statistical and Network Theory Analysis. Chemical shift data for the WT NBD1 $\triangle \mathrm{RI} \triangle \mathrm{RE}: \mathrm{VX}-809$ titration were used to analyze correlations between changes in residues from disparate regions within NBD1. Two sets of HSQC titration data were collected as follows: (WT NBD1 $\triangle \mathrm{RI} \triangle \mathrm{RE}$ : VX-809+DMSO) and (WT NBD1 $\triangle \mathrm{RI} \triangle \mathrm{RE}$ : DMSO). The data were first corrected for the presence of DMSO from the concentrated VX-809 stock. The $\omega_{1 \mathrm{H}}$ chemical shifts for the DMSO control were subtracted from the $\omega_{1 \mathrm{H}}$ for the VX-809 binding data and the same for the $\omega_{15 \mathrm{~N}}$ values, as follows:

$$
\begin{gathered}
\omega_{1 H}=\omega_{1 H, V X 809+D M S O}-\omega_{1 H, D M S O} \\
\omega_{15 N}=\omega_{15 N, V X 809+D M S O}-\omega_{15 N, D M S O}
\end{gathered}
$$

These data were analyzed for correlations separately in each dimension ( $P$ values $P_{1 \mathrm{H}}$ and $P_{15 \mathrm{~N}}$ ), which increases the amount of information extracted from the data (Dawson et al., 2013). The two independent tests of inter-residue correlation were combined using a Fisher test (Fisher, 1932). The combined Fisher test $P$ value $\left(P_{\mathrm{F}}\right)$ gives the significance of the correlation with inter-residue correlations with $P_{\mathrm{F}} \leq 0.025$ considered to be significant.

The interpretation of these correlations in terms of physical binding events is not immediately intuitive. Noise, both from the NMR experiments and the statistical analysis, further muddles interpretation. We used network theory analysis to clarify both issues. Residues with a large number of inter-residue correlations should be more important to the allosteric network of NBD1. One method of quantifying this is to calculate the degree (Diestel, 2005) and the number of inter-residue correlations for each residue.

The uncertainty in degree was estimated using the degree probability distribution (Newman et al., 2001), as follows:

$$
\begin{gathered}
P(k)=\left(\begin{array}{c}
n-1 \\
k
\end{array}\right) \rho^{k}\left(1-\rho^{k}\right)^{n-1-k} \\
P(k) \rightarrow \frac{(n \rho)^{k}}{k !} \exp (-n \rho) \text { for large } n .
\end{gathered}
$$

In the context of this analysis, $n$ is the number of residues in the network ( $n=137$ residues analyzed from VX-809:NBD1 binding data). $k$ is the degree of a residue, and $\rho$ is the probability of randomly getting a significant $\left(P_{\mathrm{F}} \leq 0.025\right)$ inter-residue correlation from the data. To estimate the value of $\rho$, the chemical shift values of the data were randomized, then the Fisher test correlation analysis was performed to find the number of inter-residue correlations predicted. This process was performed 30 times, and, on average, the probability of finding an inter-residue correlation from the randomized data was $\rho=0.081$. The probability distribution curve was calculated for degree values $k=1-25$. By taking the cumulative sum under the curve, we found that $98.6 \%$ of the randomly generated correlations were from residues with degrees $\leq 18$ for the VX-809:NBD1 binding data (Supplemental Fig. 3A). Therefore, a degree of 18 was used as a threshold in Fig. 3B and Supplemental Fig. 3B to separate residues that are important to the allosteric network from those whose appearance in the network is due to statistical uncertainties.

Similarly, network theory analysis was also applied to CL4:WT NBD1 $\Delta$ RI $\Delta$ RE titration data, originally analyzed for Fisher test correlations in the study by Dawson et al. (2013). The data set contains $n=161$ residues and, as with the VX-809:WT NBD1 $\Delta$ RI $\Delta$ RE data, the probability of observing a correlation from the randomized data was $\rho=0.081$. The degree of probability distribution was generated and summed as before, and it was found that $97.8 \%$ of the randomly generated correlations had a degree $\leq 20$, which set the uncertainty threshold for the data (Fig. 3C).

Similarities between the CL4 and VX-809 networks were analyzed using Network Analysis Tools (Brohee, 2012). The network comparison tool of this program calculates the probability that two networks are statistically independent. We compiled lists of residue pairs with significant $\left(P_{\mathrm{F}} \leq 0.025\right)$ correlations for CL4 and VX-809 titrations, respectively. Two hundred fifty correlated residue pairs (and $124 \mathrm{com}-$ mon residues) were found in both networks, and the probability of randomly finding at least 250 common correlations was calculated to define the dependence of the two networks. The list of 250 common correlation pairs was also used to estimate the importance of each residue.

\section{Results}

VX-809 Reduces Helicity of $\mathrm{H8}$ and $\mathrm{H9}$ in the C-Terminus of WT and F508del NBD1 $\triangle$ RI $\Delta$ RE. To determine whether the VX-809 compound binds to WT and/or F508del NBD1 $\triangle \mathrm{RI} \triangle \mathrm{RE}$ and to monitor any resulting conformational changes, we recorded NMR spectra at different apparent concentrations of VX-809 (note that due to the limited solubility of VX-809, concentrations are only apparent). Our NBD1 construct is composed of CFTR residues 387646, with the disordered RI (amino acids 405-436) deleted. Figure 1A shows spectral overlays for WT NBD1 $\triangle \mathrm{RI} \triangle \mathrm{RE}$ at $250 \mu \mathrm{m}$ in the apo state and after titrations of VX-809 at 1:1, 2: $1,3: 1,4: 1,5: 1$, and 6:1 apparent compound/protein molar ratios. Arrows point to a subset of peaks that shift upon the addition of VX-809. Figure 1B shows almost identical chemical shift changes upon the addition of VX-809 to F508del NBD1 $\triangle \mathrm{RI} \triangle \mathrm{RE}$. Based on the assignment of WT (Chong et al., 2015) and F508del NBD1 $\triangle \mathrm{RI} \triangle \mathrm{RE}$ (Hudson et al., 2012) resonances, we can identify the peaks having clear chemical shift changes as including D639, S641, L644, M645, and G646.

We next mapped the chemical shift changes onto a ribbon diagram of the structure of WT NBD1 $\triangle$ RI $\triangle$ RE [Protein Data Bank (PDB) identification (ID): 2PZE] (Atwell et al., 2010) for both WT (Fig. 1C) and F508del (Fig. 1D) to gain a structural perspective on the location of the changes. NBD1 is composed of an $\alpha$-subdomain (site of F508), a $\beta$-subdomain, and a central ATP-binding core (Supplemental Fig. 2B) (Lewis et al., 2004; Atwell et al., 2010). The ATP-binding core is dominated by a central $\beta$-sheet (comprising $\beta$-strands S6, S7, S8, S3, S9, and S10). Two short helices, H8 and H9, at the C-terminus fold onto $\beta$-strands S3, S9, and S10. For both WT and F508del NBD1 $\triangle \mathrm{RI} \triangle \mathrm{RE}$ the largest shifts are in helices H8 and H9, but significant shifts can also be noted in $\beta$-strands S3, S9, and S10, with very small shifts in the CL4 binding cleft. The linearity and direction of chemical shift changes upon titration (toward $\sim 8.0 \mathrm{ppm}$ ) are suggestive of a loss of helical structure in $\mathrm{H} 8$ and H9, which exist in a helix-coil equilibrium even in the absence of compound (Hudson et al., 2012). These spectral changes upon VX-809 binding are very similar to those previously shown for another compound, CFFT-001 (Hudson et al., 2012). We propose that VX-809 similarly binds to the hydrophobic surface formed by the $\beta$-strands, leading to a disruption of helices H8 and H9. Although several studies have suggested that VX-809 may bind to the NBD1:CL4 interface (Serohijos 
A

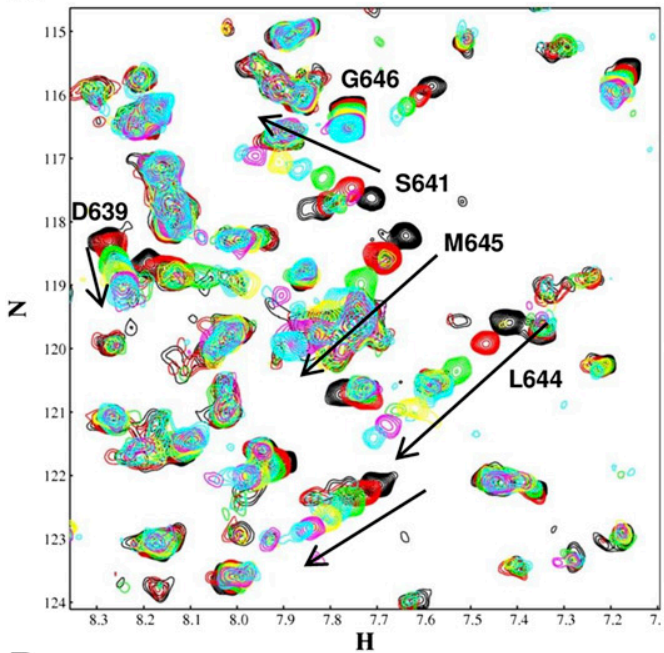

B

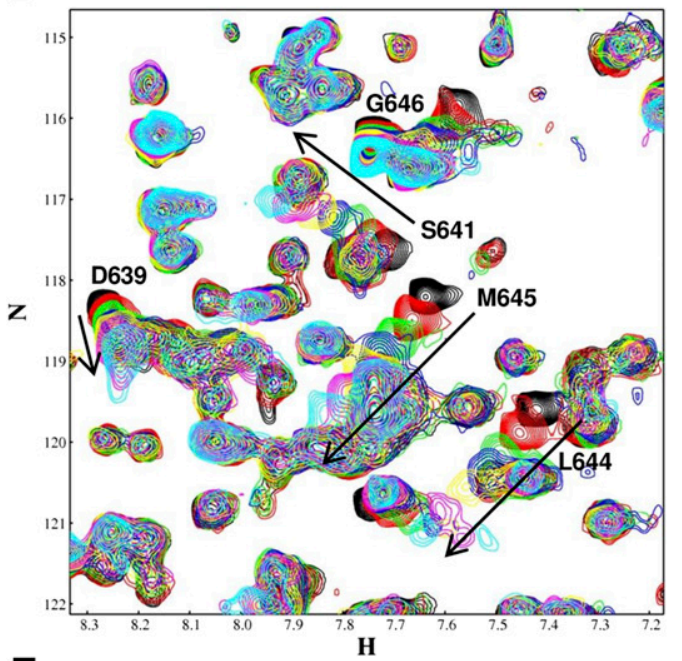

E

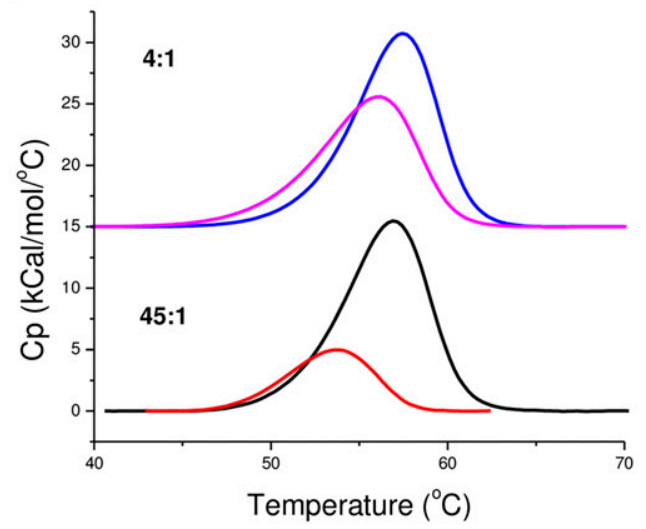

C

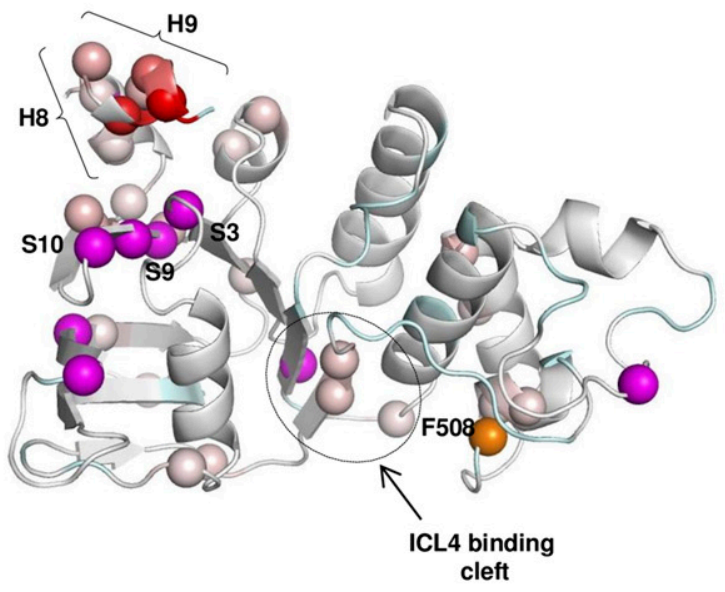

D

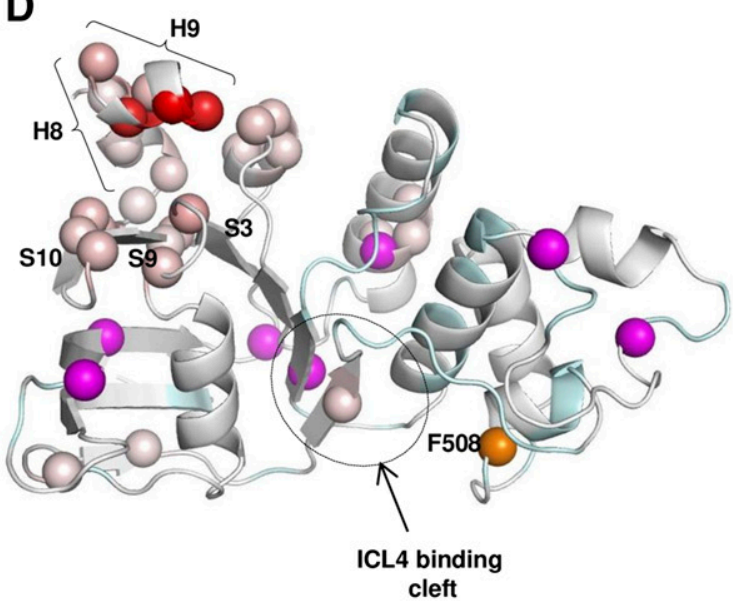

F

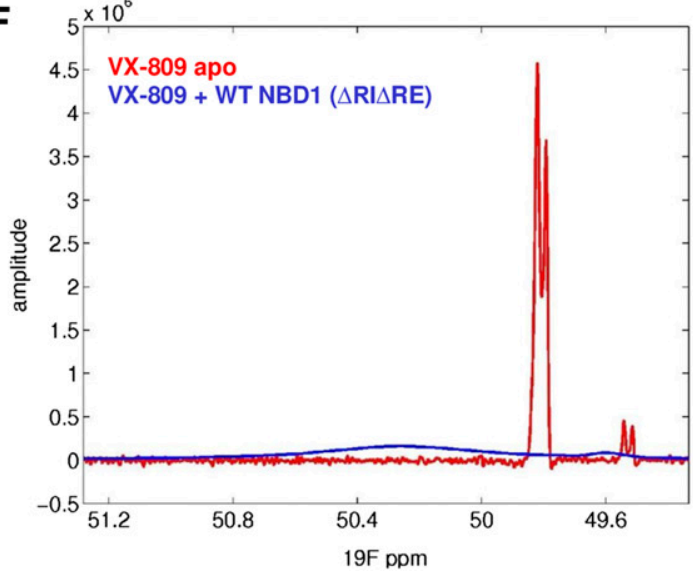

Fig. 1. VX-809 reduces helicity of $\mathrm{H} 8$ and $\mathrm{H} 9$ in the C-terminus of WT and F508del NBD1 $\triangle \mathrm{RI} \triangle \mathrm{RE}$. (A) and (B) Overlays of ${ }^{15} \mathrm{~N}-{ }^{1} \mathrm{H}$ correlation spectra at $500 \mathrm{MHz}$ for $250 \mu \mathrm{m}$ WT and F508del NBD1 $\Delta \mathrm{RI} \Delta \mathrm{RE}$, respectively, in the absence (black; background) and presence of titration points of VX-809 (1:1, red; 2:1, green; 3:1, cyan; 4:1, yellow; 5:1, magenta; 6:1, cyan). Arrows in (A) and (B) are paired with the assigned peak (where known) and indicate the subset of peaks that shift upon addition of compound and show the direction of chemical shift changes in the titration. (C) and (D) Ribbon diagrams of WT and F508del NBD1 $\triangle$ RI $\triangle$ RE (PDB ID: 2PZE), respectively, mapping chemical shift changes shown in (A) and (B). Unassigned residues are shown in cyan, and assigned residues that do not shift upon compound addition are shown in light gray. The $\mathrm{N}$ atoms for residues that show chemical shift changes are shown as spheres colored with a linear gradient from light pink to red, where light pink corresponds to the smallest shifts (beginning at $10 \mathrm{~Hz}$ ), and red to the largest shifts. Magenta spheres represent $\mathrm{N}$ atoms for residues whose chemical shift changes upon the addition of VX-809 yet are not included in the linear gradient as their post-VX-809-shifted position cannot be known with certainty. F508 is shown as an orange sphere, and the CL4 binding cleft is shown as indicated. (E) DSC traces for WT NBD1 $\triangle$ RI $\Delta$ RE at concentrations of 4:1 (blue/magenta) and 45:1 (black/orange) compound/protein ratio. (VX-809 concentrations are apparent only due to limited solubility.) The blue and black lines represent the absence of compound, and the magenta and orange lines represent the addition of compound. (F) Overlay of 1D-fluorine NMR spectra of VX-809 with (blue) and without (red) WT NBD1 $\Delta$ RI $\Delta$ RE. Spectral processing was the same in each case except that line broadening was set to 2 and 0.2 , respectively, due to the huge difference in the observed line broadening. 
et al., 2008; He et al., 2013; Okiyoneda et al., 2013), allostery between the C-terminal region (including helices H8/H9 and $\beta$-strands S3/S9/S10) and the CL4 binding site has previously been observed based on NMR data (Dawson et al., 2013).

DSC was used to confirm the binding of VX-809 to NBD1. The data in Fig. 1E illustrate the direct binding of VX-809 to WT NBD1 $\triangle \mathrm{RI} \triangle \mathrm{RE}$, as indicated by a reduction in $\mathrm{T}_{\mathrm{m}}$ of $\sim 3.1^{\circ} \mathrm{C}$ when incubated with $1.0 \mathrm{mM}$ compound (apparent concentration). This is diagnostic of VX-809 interacting with a conformation of NBD1 that is less thermostable, which is consistent with the release of the C-terminal helices $\mathrm{H} 8$ and H9 from the core. Notably, using a compound/protein ratio of $4: 1$ (1.0 mM:230 $\mu \mathrm{M})$, as in NMR experiments, showed a smaller reduction in $\mathrm{T}_{\mathrm{m}}\left(\sim 1.3^{\circ} \mathrm{C}\right.$, compare blue/magenta curves) than a ratio of $45: 1(1.0 \mathrm{mM}: 22 \mu \mathrm{M})\left(\sim 3.1^{\circ} \mathrm{C}\right.$, compare black/ orange curves). This may be attributed to the limited solubility of VX-809 and an inability to reach saturation at the concentrations required for NMR.

We next set out to observe the changes in VX-809 NMR spectra in response to NBD1 binding. 1D-Fluorine NMR experiments were recorded on $1.5 \mathrm{mM}$ VX-809 in the presence and absence of $500 \mu \mathrm{m}$ WT NBD1 $\triangle \mathrm{RI} \Delta \mathrm{RE}$. The fluorine NMR spectra (Fig. 1F) contain two peaks with narrow linewidths ( $5 \mathrm{~Hz}$ ), each split by an $8 \mathrm{~Hz}$ coupling. The addition of NBD1 to VX-809 causes these peaks to shift downfield and severely broaden, further confirming that NBD1 binds to VX-809. The chemical shift changes and broadening are due to the greatly increased correlation time as a result of the larger combined molecular weight of the NBD1 complex.

Specificity of VX-809 Binding. Because of the close functional and structural relationship between NBD1 and NBD2, we also examined potential spectral changes of $250 \mu \mathrm{M}$ NBD2 upon the addition of VX-809 (4:1) (Fig. 2A). Although NBD2 has a similar overall fold onto NBD1 and contains a hydrophobic surface patch similar to the patch formed by $\beta$-strands S3, S9, and S10 of NBD1 (PDB ID: 3GD7), chemical shift perturbations in this region or any other due to VX-809 are not detected, suggesting specificity of VX-809 binding for NBD1. To further address the specificity of spectral changes, we examined F508del NBD1 $\triangle \mathrm{RI} \Delta \mathrm{RE}$ upon the addition of two other compounds, an inactive analog of VX-809 with minimal activity $\left(\mathrm{EC}_{50}>20 \mu \mathrm{M}\right.$ ), VRT-528 (Fig. 2B), and BIA (Fig. 2C), a known NBD1-binding compound. No chemical shift changes could be detected upon titration of VRT-528 in quantities similar to those used with VX-809, strongly suggesting a lack of (or weaker) interaction between the inactive compound and the protein. Importantly, VRT-528 showed similar or better solubility, so the lack of binding is not due to more limited availability of the compound. In contrast, the addition of BIA causes spectral changes that differ significantly from those seen with VX-809. Mapping of these chemical shift changes onto a ribbon diagram of the structure of WT NBD1 $\triangle \mathrm{RI} \triangle \mathrm{RE}$ (PDB ID: 2PZE) show that the largest shifts are in the $\alpha$-subdomain, which is consistent with previous reports (Hall et al., 2015; He et al., 2015) (Fig. 2D). In agreement with their different binding sites, VX-809 and BIA show elevated responses when used together (He et al., 2015) on full-length CFTR. Also noteworthy, and in contrast to the situation with VX-809, is the fact that BIA shows a positive $\mathrm{T}_{\mathrm{m}}$ shift upon binding to NBD1. Overall, these results argue that VX-809 binds specifically to the patch formed by $\beta$-strands S3, S9, and S10 and likely has a mechanism of action distinct from BIA.
Poor VX-809 Solubility Leads to a Low Apparent Binding Affinity for NBD1. The concentration dependence of VX-809 aggregation in NMR buffer is shown using DLS (Supplemental Fig. 4, A and B). Note that after filtering a $1 \mathrm{mM}$ sample, the DLS intensity dropped to the same level as the buffer and the estimated particle size was the same as the buffer $(\sim 0.4 \mathrm{~nm}$, less than $1 \mathrm{kDa})$, suggesting that a significant portion of VX-809 forms large aggregates at concentrations greater than $0.06 \mathrm{mM}$. The narrow linewidths of our fluorine VX-809 data indicate that the aggregated VX-809 is in exchange with a soluble fraction that is able to bind to NBD1. Thus, VX-809 likely resides within an equilibrium of the following three populations: highly aggregated, monomeric (free and soluble), and NBD1 bound, where the aggregated population far outweighs the others at NMR concentrations (Supplemental Fig. 4C). The changes in chemical shift data for residues S624, Y627, S635, D639, S641, L644, M645, and G646, some of which are shown in Fig. 1, were fit together to calculate approximate apparent affinities $\left(K_{\mathrm{d}}\right.$ values $)$ of $1.3 \mathrm{mM}( \pm 0.4)$ and $3.2 \mathrm{mM}( \pm 1.5)$ for WT and F508del NBD1 $\triangle$ RI $\triangle$ RE, respectively (Supplemental Fig. 5, A and $B$ ). Since NMR data were obtained using concentrations well above $0.06 \mathrm{mM}$, these titrations overestimate the amount of VX-809 in solution and thus likely underestimate the affinity of this compound for NBD1. VX-809 insolubility under NMR conditions together with cellular environments that may localize or even concentrate small molecules could potentially address the significant difference in apparent affinity to the $\mathrm{EC}_{50}$ values, $0.1 \pm 0.1$ and $0.5 \pm 0.1 \mu \mathrm{M}$ (based on F508del-CFTR maturation and chloride transport, respectively) observed in Fischer rat thyroid cells (Van Goor et al., 2011).

Correlation Analysis of Ligand:NBD1 Binding Data Demonstrates that VX-809 and CL4 Probe the Same Allosteric Network. In addition to the relatively large chemical shift changes at the C-terminal $\beta$-strands S3, S9, and S10 and helices H8/H9 (collectively called the C-terminal region), we also observed smaller chemical shift changes at the CL4 cleft. Previous data in our laboratory (Dawson et al., 2013) have shown an allosteric coupling between these two regions as well as the $\mathrm{RI}$ deletion site. Taken together with the biophysical data above, we considered the possibility of VX-809 probing a similar allosteric network.

To determine whether the small chemical shift changes within the CL4 binding cleft are significant and due to VX-809 binding at the C-terminal $\beta$-strands, we did a correlation analysis (Fisher, 1932). Two residues that are affected by the same binding event should have correlated chemical shift changes (Selvaratnam et al., 2011; Dawson et al., 2013). A "contact map" shows the significant $\left(P_{\mathrm{F}} \leq 0.025\right)$ inter-residue correlations due to VX-809 binding (Fig. 3A). Residues near the C-terminal region and the CL4 binding cleft appear to have a relatively high number of inter-residue correlations. Residues with a large number of inter-residue correlations should be more important to the allosteric network of NBD1. One method of quantifying this is to calculate the degree (Diestel, 2005), the number of inter-residue correlations for each residue, using network theory analysis (see Materials and Methods). We separated residues that are important to the allosteric network from those whose appearance in the network is due to statistical uncertainties (identifying a degree threshold of 18) and mapped the degree values onto 
A

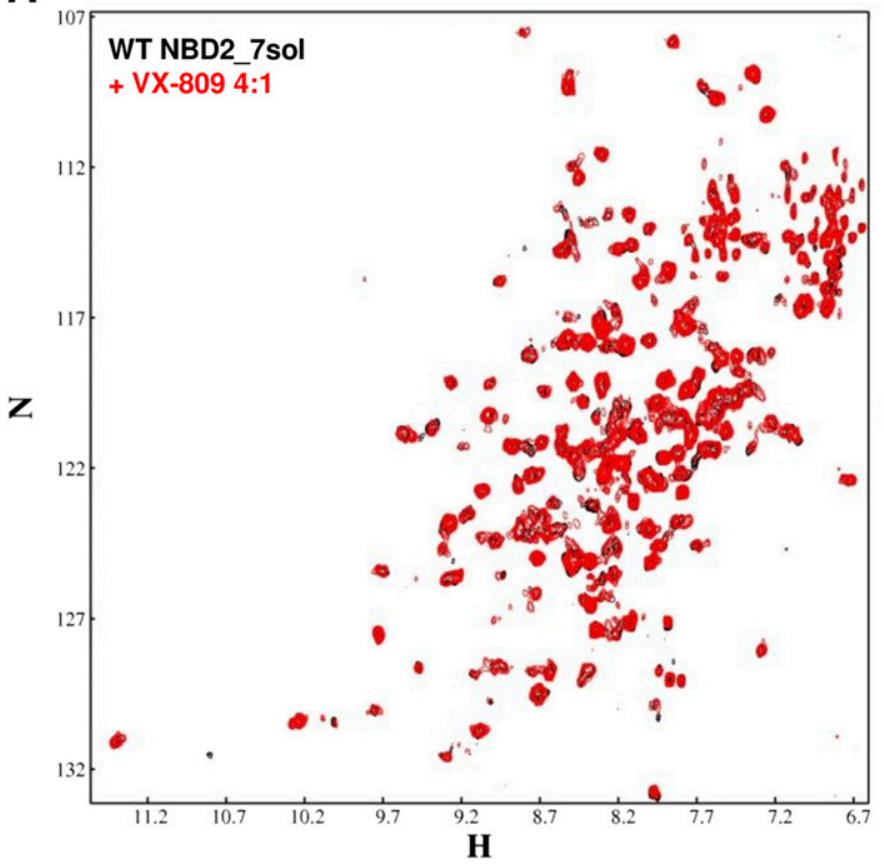

B

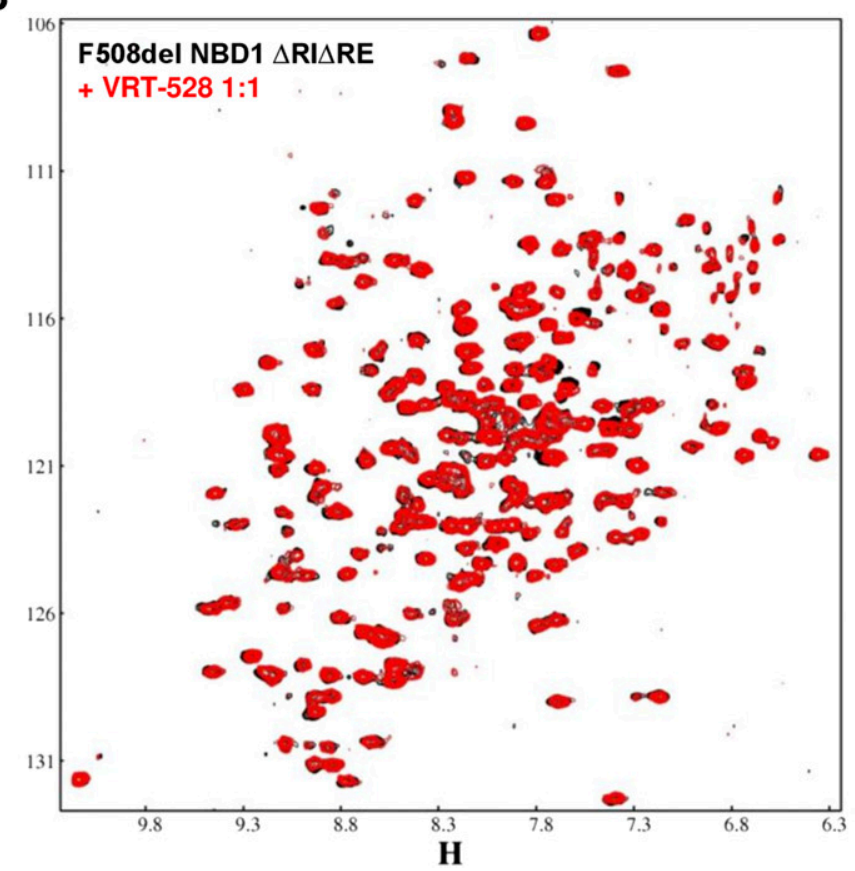

C

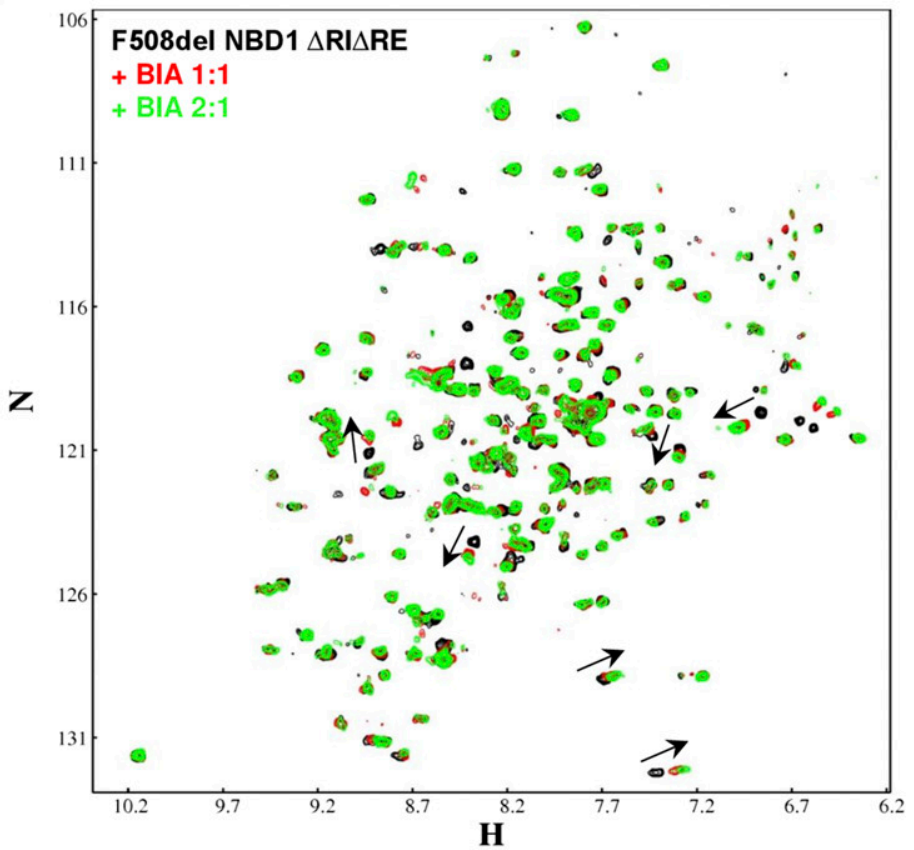

D

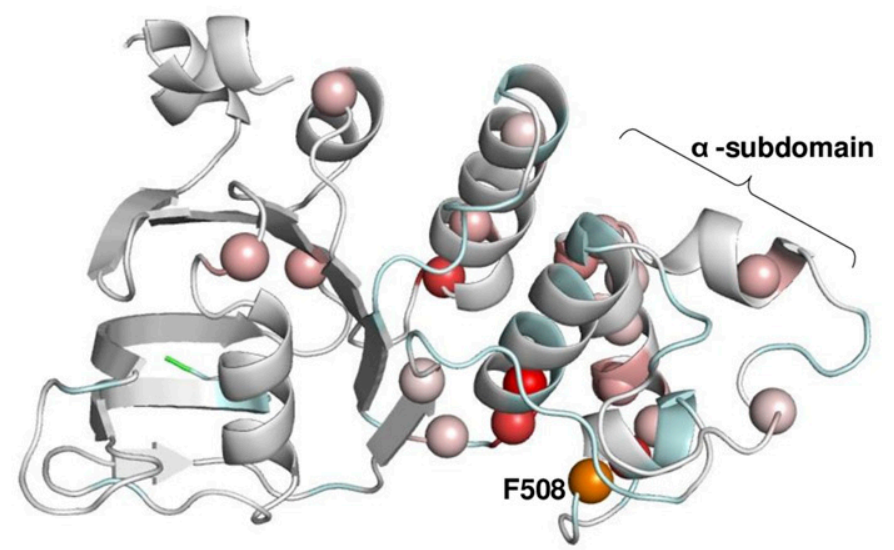

Fig. 2. NBD2, VRT-528, and BIA as controls for VX-809 binding. Overlays of ${ }^{15} \mathrm{~N}-{ }^{1} \mathrm{H}$ correlation spectra at $500 \mathrm{MHz}$ in the absence (black, background) and presence (red, foreground) of VX-809 for $250 \mu \mathrm{m}$ NBD2 (A) or of VRT-528 (B) and BIA (C) for F508del NBD1 $\triangle$ RI $\triangle$ RE. Note the absence of chemical shift changes in (A) and (B) and the subset of peak shift changes in (C) (arrows) that differs from those seen upon a VX-809 titration in NBD1. (D) Ribbon diagrams of WT NBD1 $\triangle$ RI $\triangle$ RE (PDB ID: 2PZE) mapping the chemical shift changes shown in (C). Coloring is as described in the legend of Fig. 1 .

the NBD1 structure (Fig. 3B). This analysis demonstrates that VX-809 binding to WT NBD1 $\triangle$ RI $\triangle$ RE perturbs the C-terminal region and $\beta$-strand $\mathrm{S} 6$ of the CL4 cleft, suggesting that these regions are a significant part of the allosteric network.

Allostery between the C-terminal region and the CL4 binding site has been previously observed upon CL4 peptide binding (Dawson et al., 2013). A similar allosteric pattern is observed here upon VX-809 binding (compare Fig. 3, B and C). This is not unexpected for two ligands probing the same allosteric network. Network theory analysis was also applied to CL4:WT NBD1 $\triangle \mathrm{RI} \Delta \mathrm{RE}$ titration data, originally analyzed for Fisher test correlations in the study by Dawson et al. (2013). Using a degree of 20 for the threshold (see Materials and Methods), it was found that residues in the C-terminal region, the CL4 cleft, and the RI deletion site are important members in the allosteric network perturbed by CL4:NBD1 binding, which is in agreement with the network previously observed by CL4 peptide binding (Dawson et al., 2013). 

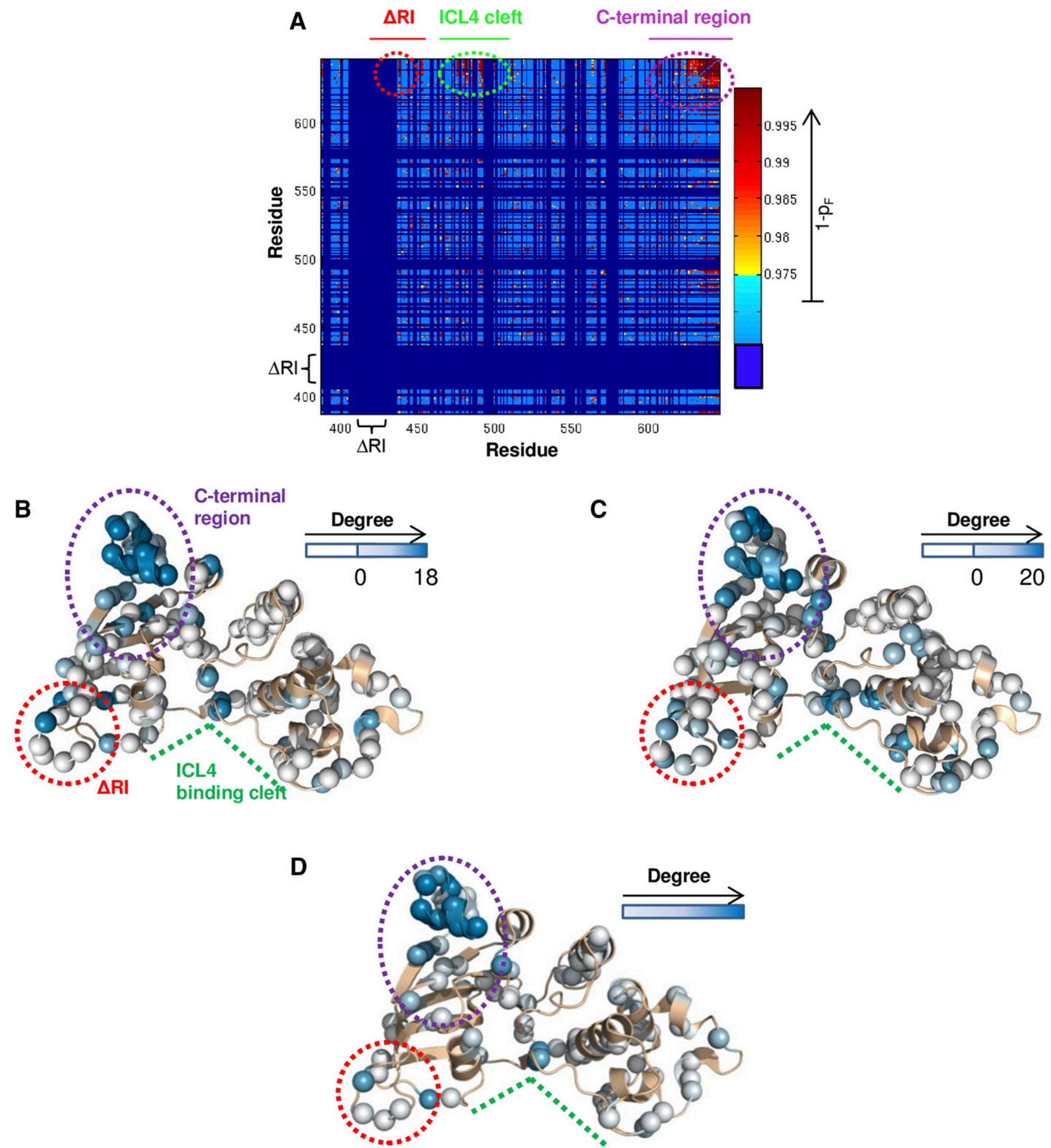

Fig. 3. (A) Inter-residue correlation contact map for VX-809:NBD1 titration. Pairs of residues with significant correlations $\left(1-P_{\mathrm{F}} \geq 0.975\right)$ are shown in a yellow-to-red heat map, with red being the most significant. Light blue indicates nonsignificant correlations, and dark blue marks the residue pairs that were not analyzed due to the lack of assignment or deletion from the construct ( $\triangle \mathrm{RI})$. The inter-residue correlations among the RI deletion site (red circle), CL4 binding cleft (green circle), and the C-terminal $\beta$-strands/helices region (purple circle) are indicated. VX-809:NBD1 (B) and CL4:NBD1 (C) degree values that are above the threshold have been mapped onto the NBD1 structure (PDB ID: 2PZE). Similar network theory analysis of the CL4:NBD1 data found that a threshold at degree $>20$ eliminated $98 \%$ of the noise. Analyzed residues are indicated as spheres with above threshold degree values colored by a white-to-blue gradient and residues below threshold are shown as white. Unassigned residues are shown as tan ribbons. VX-809:NBD1 and CL4: NBD1 data have similar patterns of mapped degree values. (D) The similarity between the sets of residues probed by VX-809 and CL4 was found to be statistically significant, implying that the ligands probe the same allosteric network. Two hundred fifty inter-residue correlations are found in common for both sets of binding data. The allosteric network coupling the C-terminal region and the CL4 binding cleft is shown by mapping these common correlations onto the NBD1 structure as degree values. 
To quantitatively demonstrate that CL4 and VX-809 probe the same allosteric network, the similarities between the two networks were analyzed using Network Analysis Tools (Brohee, 2012). We compiled lists of residue pairs with significant $\left(P_{\mathrm{F}} \leq 0.025\right)$ correlations for CL4 and VX-809 titrations, respectively. Two hundred fifty correlated residue pairs (and 124 common residues) were found in both networks. The $P$ value is $1.2 \times 10^{-18}$, meaning that the probability of randomly finding at least 250 common correlations is nearly zero, suggesting that the two networks are dependent. We also used the list of 250 common correlation pairs to estimate the importance of each residue (Supplemental Fig. 3C). The residues with the greatest degree values are found in the C-terminal region of NBD1, in the CL4 binding site (especially residues 490-492), and in the RI deletion site (Fig. 3D). Using these quantitative tools, we have demonstrated via NMR spectroscopy and statistical analysis that ligands CL4 and VX-809 probe the same allosteric network.

Docking of VX-809 onto the NBD1 Crystal Structure. To explore the structural means of VX-809 binding to the C-terminal $\beta$-strand site, as defined by our NMR data, we computationally docked the compound onto NBD1 (Fig. 4). To mimic the destabilization of helices $\mathrm{H} 8$ and $\mathrm{H} 9$ required for VX-809 binding, the coordinates of the $2 \mathrm{PZE}$ crystal structure were truncated at residue 630 , exposing the proposed VX-809 binding pocket. The docking process generated nine different docked VX-809 conformations with similar predicted binding energies and some common features are observed in all conformations. In all cases, VX-809 lies along strand S9 and is sandwiched by F626 on strand S10, and M607 and L610 on helix H7. In all docked structures, F626 is involved in a stacking interaction with the compound. In six of the nine cases, F626 stacks against the 2,2-difluoro-1,3-benzodioxol portion of VX-809 (Fig. 4A). In the remaining three cases, F626 stacks against the benzoic acid portion of VX-809 (Fig. 4B). In most cases, I618 forms hydrophobic contacts with VX-809. Additional interactions can be observed between VX-809 and H620 and Y625 in some of the docked structures. Note that this docking was constrained to a region around those residues identified by chemical shift analysis, whereas docking in the absence of data could suggest numerous other potential interacting sites with dubious relevance. These docking studies suggest that the proposed binding pocket is a reasonable location for compound binding, which would then sterically inhibit the interaction of $\mathrm{H} 8$ and $\mathrm{H} 9$ with the NBD1 core and lead to helix destabilization.

An H620D Variant Attenuates VX-809 and CFFT-001 Binding to NBD1. To address the specificity of $\beta$-strands S3/S9/S10 as the binding site for VX-809, as well as CFFT-001, a previously published compound sharing similar binding data with VX-809 (Hudson et al., 2012), we used our docking model to identify residues that upon mutation may interfere with VX-809 binding. We noted that the carboxylate of VX-809 was in close proximity to residues $\mathrm{S} 459$ (in the loop between S3 and S9) and H620 (in S9) in some conformations of the docked structures. We predicted that an acidic residue at one or both of these residues could repel the carboxylate of VX-809, thereby attenuating binding. Figure 5A shows a spectral overlay of WT NBD1 $\triangle \mathrm{RI} \triangle \mathrm{RE}$ and H620D NBD1 $\triangle \mathrm{RI} \Delta \mathrm{RE}$ to demonstrate an identical overall fold. The few peaks that do shift upon mutation and can be identified from assignment data are labeled. As expected, the residues immediately neighboring H620, E621, and L619 are the most affected and show the largest shifts (arrows). The majority of peaks affected by the mutation are those found in helices H8 and H9 (Q634, N635, D639, M645, and G646) with several others in $\beta$-strands S3/S9/S10 (G458, L619, E621, G622, and Y627) and one in the $\beta$-subdomain (N445). In addition, there are several peaks that move and new peaks that appear that cannot be identified with certainty (asterisk) since they are not assigned. Spectral overlays of H620D NBD1 $\triangle \mathrm{RI} \triangle \mathrm{RE}$ in the apo state and after titrations of 1:1, 2:1, and 3:1 apparent molar ratios of VX-809 and CFFT-001 to protein demonstrate chemical shift changes for only two peaks (unassigned, asterisks in Fig. 5B) for VX-809 and no chemical shift changes for CFFT-001 (Fig. 5C). Comparison of the VX-809 and CFFT-001 titrations with those seen in Fig. 1A and in Hudson et al. (2012), respectively, shows that peak shifts are far less numerous and significantly smaller. Thus, H620D has a significant effect on VX-809 and CFFT-001 binding, strongly supporting the region around H620, $\beta$-strands S3/S9/S10, as the binding site for these compounds on NBD1.

Effect of H620D in Full-Length CFTR on Correction by VX-809 and CFFT-001. Since NMR data strongly indicate that at least one target of VX-809 binding is on $\beta$-strands S3/S9/S10 of NBD1, similar to CFFT-001, we addressed whether correction by VX-809 and CFFT-001 within the context of full-length CFTR was impeded by the H620D variant. HEK 293 cells stably expressing either F508del-CFTR or F508del-H620D-CFTR were incubated with VX-809 or CFFT-001, and the degree of correction/processing was compared by analysis of total Band C (Fig. 5E). The H620D mutation decreases the level of correction of F508del CFTR by both VX-809 and CFFT-001, as reflected by the lower amount of Band C. Although having data from a single experiment using one specific dose makes it challenging to deconvolute the effect of DMSO from the H620D mutant, a
A

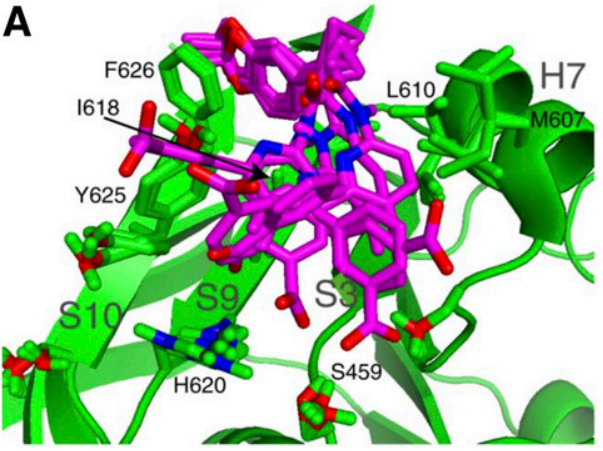

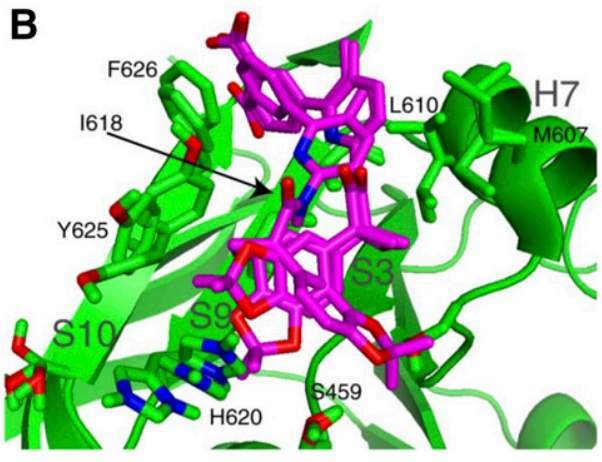

Fig. 4. Ribbon diagrams of the S3, S9, and $\mathrm{S} 10$ region of NBD1 with VX-809 docked. NBD1 and VX-809 are shown in green and violet, respectively. Nitrogen, oxygen, and fluorine atoms are shown in blue, red, and cyan, respectively. VX-809 docked in two main orientations. The VX-809 conformers in the dominant docking orientation, which was present in six of nine of the lowest energy conformers, are shown in (A). The remaining three low-energy VX-809 conformers in the secondary docking orientation are shown in (B). 
A

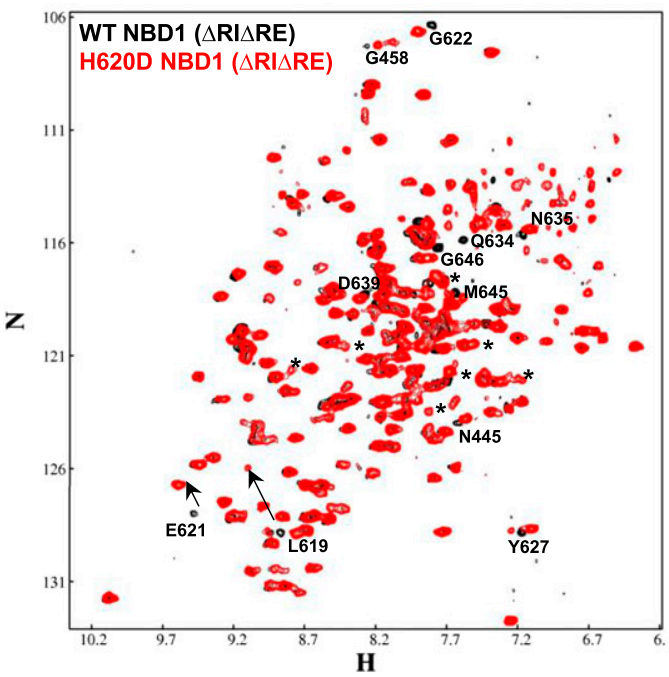

C

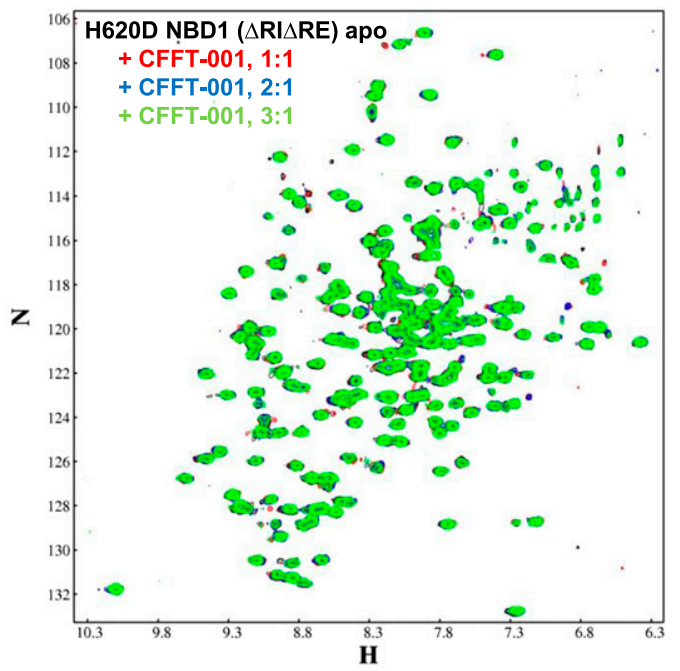

E

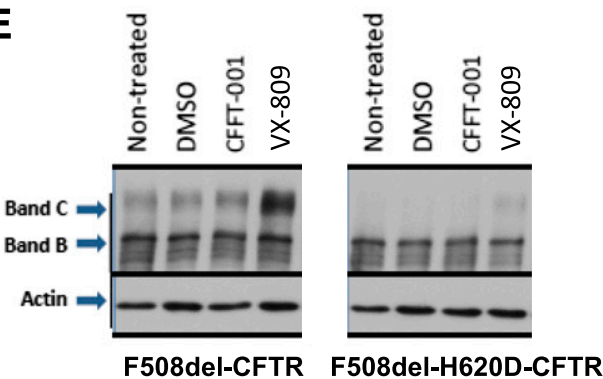

B

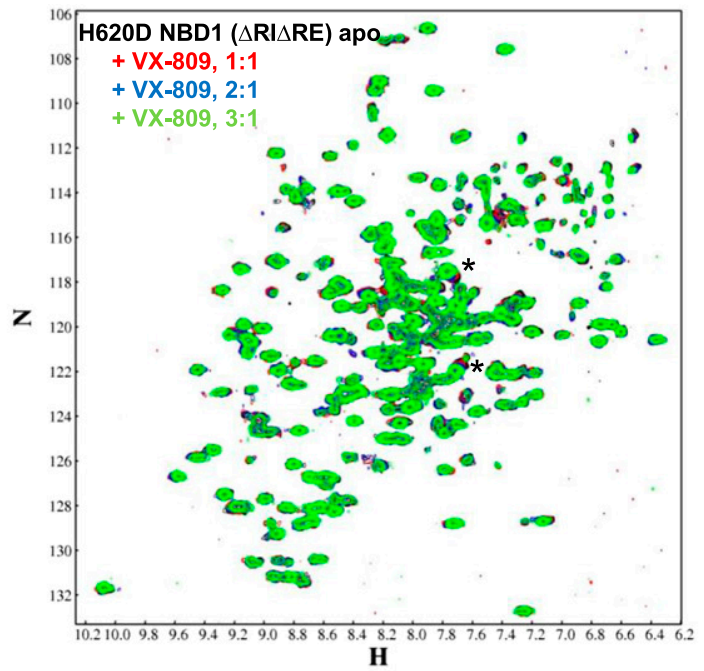

D

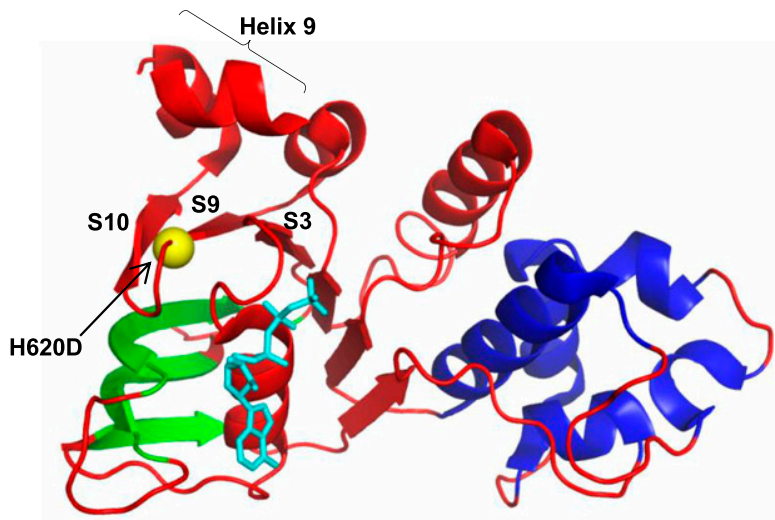

F

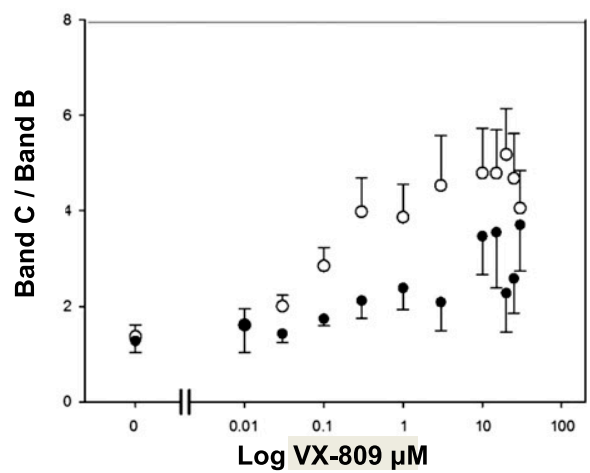

Fig. 5. H620D abrogates binding of VX-809 to WT NBD1 $\Delta$ RI $\Delta$ RE. Overlay of ${ }^{15} \mathrm{~N}-{ }^{1} \mathrm{H}$ correlation spectra at $500 \mathrm{MHz}$ for $250 \mu \mathrm{m}$ WT NBD1 $\Delta$ RI $\Delta$ RE (black, background) and H620D NBD1 $\Delta \mathrm{RI} \Delta \mathrm{RE}$ (red, foreground) (A); $250 \mu \mathrm{m}$ H620D NBD1 $\Delta \mathrm{RI} \Delta \mathrm{RE}$ in the absence (black; background) and presence of titration points (1:1, red; 2:1, blue; 3:1, green) of VX-809 (B) and CFFT-001 (C). Note the absence of chemical shift changes. (D) Ribbon diagram of WT NBD1 $\triangle$ RI $\triangle$ RE (PDB ID: 2PZE) where the N atom of the H620 is shown as a yellow sphere. Coloring is as described in Supplemental Fig. 1B. (E) HEK-293 cells expressing F508del-CFTR or F508del-H620D-CFTR were treated with $1 \mu \mathrm{m}$ VX-809 or CFFT-001 and were incubated for 24 hours at $30^{\circ} \mathrm{C}$. CFTR correction by VX-809 and CFFT-001, as shown by Band C, is reduced by the H620D mutation. (F) Quantitative analysis ( $n=6$ ) of HEK-293 cells expressing F508del-CFTR $(\bigcirc)$ or F508del-H620D-CFTR $(\bullet)$ and incubated with increasing concentrations of VX-809 demonstrate the suppression, but not complete abrogation, of correction.

quantitative analysis of data for multiple doses of VX-809 $(n=6)$ clearly shows the suppression of correction due to the mutation (Fig. 5F). This is consistent with VX-809 binding to NBD1 being a component of its cellular mechanism of action. As expected, complete abrogation of VX-809 corrector activity was not observed since another direct binding site of VX-809 in 
full-length CFTR, MSD1 (Ren et al., 2013), remains intact and has not been mutated.

\section{Discussion}

The mechanism by which VX-809 exerts its corrective therapeutic action on F508del CFTR is not well understood. Based on its ability to restore thermostability to purified protein (Ford et al., 2014) it has been proposed that VX-809 likely has a direct interaction with F508del CFTR. This is consistent with studies showing two VX-809 derivatives binding to F508del CFTR (Sinha et al., 2015) and limited proteolysis studies showing the effect of VX-809 protection on NBD1-dependent MSD1 folding (Ren et al., 2013). We report here, for the first time, direct binding of VX-809 to human WT and F508del NBD1 $(\triangle \mathrm{RI} \triangle \mathrm{RE})$. The binding leads to conformational changes within NBD1 that allosterically couple the binding site, the NBD1:CL4 interface, and possibly the NBD dimerization surface.

Within the context of an isolated NBD1 construct, we show the site of VX-809 binding is $\beta$-strands $\mathrm{S} 3, \mathrm{~S} 9$, and $\mathrm{S} 10$. Binding to the $\beta$-strands leads to a displacement of helices H8 and H9 from the core of the molecule. The specificity of binding is supported by the abrogation of binding in the H620D mutation, a residue in $\beta$-strand S9, and the lack of binding to the $\beta$-strands of NBD2. The helices (H8 and H9), which are displaced by VX-809, are destabilized to a coil conformation and likely are not involved in binding to VX-809. The displacement of $\mathrm{H} 8$ and $\mathrm{H} 9$ is supported by DSC data showing a reduction in $\mathrm{T}_{\mathrm{m}}$ upon ligand binding. Although one would typically expect compound binding to result in an apparent increase in $T_{m}$ and stability, the negative $T_{m}$ shift we observe indicates binding to a non-native state, which is consistent with binding to the destabilized conformation where the helices are released from the core. This supports and reinforces the interpretation of the changes observed by NMR. The conformational changes and $\mathrm{T}_{\mathrm{m}}$ reduction upon VX-809 binding are nearly identical to those previously described for CFFT-001 (Hudson et al., 2012) but differ greatly from changes observed for BIA (He et al., 2015), suggesting the two compounds VX-809 and CFFT-001 may share some part of their mechanism of action that is distinct from BIA.

In addition to the large chemical shift changes at the $\beta$-strands upon VX-809 binding, we also detect smaller chemical shift changes at the NBD1:CL4 interface. We have previously shown evidence for an allosteric network within NBD1 that connects the CL4 binding cleft to two other regulatory sites in NBD1, the RI deletion site, and the NBD1 C-terminal site composed of $\beta$-strands S3, S9, and S10 and helices H8/H9 (Dawson et al., 2013). We propose, based on NMR data and inter-residue correlation analysis, that VX-809 binds directly to $\beta$-strands S3, S9, and S10, and perturbs residues in the CL4 binding site via allosteric interactions (consistent with the much smaller chemical shift changes at the CL4 interface upon binding), possibly stabilizing the NBD1:CL4 interface. Additionally, a statistical analysis demonstrates that the VX-809 compound and a CL4 peptide (Dawson et al., 2013), while binding to different sites within NBD1, probe the same allosteric network. Part of this allosteric network, between the $\alpha$-subdomain making up part of the CL4 binding cleft and the $\beta$-subdomain containing the RI, has also been elucidated via molecular dynamics and network theory analysis (Aleksandrov et al., 2012; Proctor et al., 2015). We propose that the binding of VX809 to the $\beta$-strands disrupts interactions between helices $\mathrm{H} 8 / \mathrm{H} 9$ and consequently the $\mathrm{RE} / \mathrm{R}$ region, thereby relieving their inhibitory effect on NBD dimerization while concurrently stabilizing NBD1:CL4 interactions via an allosteric mechanism.

Several studies have suggested that VX-809 may bind directly to the NBD1:CL4 interface (Serohijos et al., 2008; He et al., 2013; Okiyoneda et al., 2013). Other studies have shown that the rescue of F508del CFTR maturation to near WT levels can be accomplished by simultaneous correction of the NBD1 thermostability defect and the disrupted NBD1: CL4 interface (Mendoza et al., 2012; Rabeh et al., 2012). Since VX-809 does not appear to stabilize isolated NBD1 (Loo et al., 2013), it has been assumed to stabilize the full-length protein by correcting the NBD1:CL4 interface defect, a proposed mechanism for mutational rescue of F508del CFTR. This is supported by docking and mutagenesis studies suggesting that VX-809 directly restores defective NBD1:CL4 interactions (He et al., 2013). In contrast, our demonstration of an allosteric effect of VX-809 on the CL4 interaction is consistent with a number of observations that are difficult to reconcile with direct binding of VX-809 to the CL4 interface. These include the fact that VX-809 corrects multiple CF-causing mutants in CL4 as well as outside of the NBD1:CL4 interface, including those found in other CLs (He et al., 2013) and MSD1 (Ren et al., 2013), suggesting that VX-809 need not specifically interact at the site of pathologic mutations. Importantly, VX-809 enhances the maturation of CFTR that has been corrected by either NBD1 stabilizing mutations or NBD1:CL4 interface stabilizing mutations (He et al., 2013). Our allostery data are also consistent with the observations that stabilizing NBD1 alone, as shown in He et al. (2015), is sufficient to correct CFTR and that the dual-target approach of additionally mutating the NBD1:CL4 interface can result in a locked open channel that is constitutively active. VX-809 is able to correct both WT and F508del CFTR (Van Goor et al., 2011). Since the NBD1:CL4 interface is substantially different in WT and F508del, this further supports the notion that VX-809 does not act at this interface but, rather, modulates this interface through an allosteric mechanism. In addition, it indicates that WT and F508del NBD1 are likely to share common allosteric pathways.

It has been reported that VX-809 acts early on in the biogenesis of CFTR and suppresses folding defects within F508del CFTR by modulating the conformation of MSD1 and increasing the efficiency of interactions between MSD1 and other domains of CFTR, including NBD1, MSD2, and NBD2 (Ren et al., 2013), and thus the allosteric network may extend to MSD1 as well. We propose that VX-809 correction may work through allosteric connections between various regions of CFTR, such that the stabilization of one region or interdomain interaction can enhance the overall stability of the channel. Although it is possible that NBD1 binding of VX-809 could exert allosteric effects throughout CFTR to account for effects in MSD1, the results of experiments (Ren et al., 2013) are consistent with direct binding to MSD1. Thus, VX-809 likely binds directly to sites in both MSD1 and NBD1. Notably, we detect only partial effects on correction in full-length CFTR where VX-809 binding has been abrogated with the H620D mutation, suggesting that the NBD1 binding site is likely a 
secondary component with the primary mechanism of action occurring at the MSD1 site. Interestingly, the level of VX-809 correction is enhanced to nearly WT levels when combined with approaches that address the instability of F508del NBD1 and defective NBD1:CL4 interactions, indicating that the action of VX-809 on MSD1 alone is not enough for complete restoration.

A final point to consider is that VX-809 has been shown to have an inhibitory effect on F508del CFTR function at high doses in Fischer rat thyroid cells (Van Goor et al., 2011). Because the VX-809 concentration used in our NMR and DSC experiments is likely to be higher than the therapeutic range, it is possible that binding to the $\mathrm{C}$-terminal $\beta$-strands is an inhibitory event and thus is not relevant to F508del CFTR rescue. However, it is not possible to know the actual concentrations of VX-809 in the cell, as lipid and other compartments may concentrate pharmaceuticals. This may be particularly significant for VX-809, which is quite hydrophobic, making even our in vitro concentration measurements unreliable. Whatever the manner in which VX-809 binding to NBD1 acts, it is important to recognize this binding as a component of its mode of action. Even if direct binding to MSD1 is the primary mechanism of correction, the observations that both VX-809 and CFFT-001 interact with the C-terminal $\beta$-strands in NBD1 and that mutation of this region reduces VX-809-mediated correction suggest that this site is an interaction hotspot that should be considered for understanding therapeutics.

\section{Acknowledgments}

The authors thank Drs. Ranjith Muhandiram, Lewis E. Kay, and Scott Prosser for assistance with NMR experiments; and Dr. Algirdas Velyvis for the scripts to calculate global fit $K_{\mathrm{d}}$ values from chemical shift changes. The authors also thank Frederick Van Goor, Vertex Pharmaceuticals, Inc., for VRT-528, the initial supply of VX-809, and stimulating discussions. In addition, thanks to J. Spencer Emtage, Hal A. Lewis, Shane Atwell, and the entire SGX Pharmaceuticals, Inc. (San Diego, CA) CFTR team for providing the unpublished crystal coordinate data for the BIA complex with NBD1.

\section{Authorship Contributions}

Participated in research design: Hudson, Dawson, Chong, Yang, Thomas, Brouillette, and Forman-Kay.

Conducted experiments: Hudson, Dawson, Chong, Yang, and Millen

Contributed new reagents or analytic tools: Thomas.

Performed data analysis: Hudson, Dawson, Chong, Yang, Millen, Brouillette, and Forman-Kay.

Wrote or contributed to the writing of the manuscript: Hudson, Dawson, Chong, Yang, Brouillette, and Forman-Kay.

\section{References}

Aleksandrov AA, Kota P, Cui L, Jensen T, Alekseev AE, Reyes S, He L, Gentzsch M, Aleksandrov LA, Dokholyan NV, et al. (2012) Allosteric modulation balances thermodynamic stability and restores function of $\Delta$ F508 CFTR. J Mol Biol 419: $41-60$.

Atwell S, Brouillette CG, Conners K, Emtage S, Gheyi T, Guggino WB, Hendle J, Hunt JF, Lewis HA, Lu F, et al. (2010) Structures of a minimal human CFTR first nucleotide-binding domain as a monomer, head-to-tail homodimer, and pathogenic mutant. Protein Eng Des Sel 23:375-384.

Bobadilla JL, Macek, Jr M, Fine JP, and Farrell PM (2002) Cystic fibrosis: a worldwide analysis of CFTR mutations-correlation with incidence data and application to screening. Hum Mutat 19:575-606.

Boyle MP, Bell SC, Konstan MW, McColley SA, Rowe SM, Rietschel E, Huang X, Waltz D, Patel NR, and Rodman D; VX09-809-102 Study Group (2014) A CFTR corrector (lumacaftor) and a CFTR potentiator (ivacaftor) for treatment of patients with cystic fibrosis who have a phe508del CFTR mutation: a phase 2 randomised controlled trial. Lancet Respir Med 2:527-538.

Brohée S (2012) Using the NeAT toolbox to compare networks to networks, clusters to clusters, and network to clusters. Methods Mol Biol 804:327-342.
Chappe V, Irvine T, Liao J, Evagelidis A, and Hanrahan JW (2005) Phosphorylation of CFTR by PKA promotes binding of the regulatory domain. $E M B O J$ 24: $2730-2740$.

Chong PA, Farber PJ, Vernon RM, Hudson RP, Mittermaier AK, and Forman-Kay JD (2015) Deletion of phenylalanine 508 in the first nucleotide-binding domain of the cystic fibrosis transmembrane conductance regulator increases conformational exchange and inhibits dimerization. J Biol Chem 290:22862-22878.

Clancy JP, Rowe SM, Accurso FJ, Aitken ML, Amin RS, Ashlock MA, Ballmann M, Boyle MP, Bronsveld I, Campbell PW, et al. (2012) Results of a phase IIa study of VX-809, an investigational CFTR corrector compound, in subjects with cystic fibrosis homozygous for the F508del-CFTR mutation. Thorax 67:12-18.

Corradi V, Vergani P, and Tieleman DP (2015) Cystic fibrosis transmembrane conductance regulator (CFTR): closed and open state channel models. J Biol Chem 290:22891-22906.

Dalemans W, Barbry P, Champigny G, Jallat S, Dott K, Dreyer D, Crystal RG, Pavirani A, Lecocq JP, and Lazdunski M (1991) Altered chloride ion channel kinetics associated with the delta F508 cystic fibrosis mutation. Nature 354:526-528.

Dawson JE, Farber PJ, and Forman-Kay JD (2013) Allosteric coupling between the intracellular coupling helix 4 and regulatory sites of the first nucleotide-binding domain of CFTR. PLoS One 8:e74347.

Delaglio F, Grzesiek S, Vuister GW, Zhu G, Pfeifer J, and Bax A (1995) NMRPipe: a multidimensional spectral processing system based on UNIX pipes. J Biomol NMR 6:277-293.

Diestel R (2005) Graph Theory, 3rd ed, Springer-Verlag, Berlin, New York.

Fisher RA (1932) Statistical Methods for Research Workers, Oliver \& Boyd, Edinburgh.

Ford B, Meng X, Wang X, and Pollock N (2014) Biophysical properties of purified CFTR containing the most common CF causing mutations and in the presence and absence of drugs. Pediatr Pulmonol 46(Suppl 38):S232.

Hall JD, Wang H, Byrnes LJ, Shanker S, Wang K, Efremov IV, Andrew Chong P, Forman-Kay JD, and Aulabaugh AE (2015) Binding screen for cftr correctors finds new chemical matter and yields insights into CF therapeutic strategy. Protein Sci 24:105-116

He L, Kota P, Aleksandrov AA, Cui L, Jensen T, Dokholyan NV, and Riordan JR (2013) Correctors of $\Delta$ F508 CFTR restore global conformational maturation without thermally stabilizing the mutant protein. FASEB $J$ 27:536-545.

He L, Aleksandrov AA, An J, Cui L, Yang Z, Brouillette CG, and Riordan JR (2015) Restoration of NBD1 thermal stability is necessary and sufficient to correct $\Delta \mathrm{F} 508$ CFTR folding and assembly. J Mol Biol 427:106-120.

Hudson RP, Chong PA, Protasevich II, Vernon R, Noy E, Bihler H, An JL, Kalid O, Sela-Culang I, Mense M, et al. (2012) Conformational changes relevant to channe activity and folding within the first nucleotide binding domain of the cystic fibrosis transmembrane conductance regulator. J Biol Chem 287:28480-28494.

Hwang TC and Sheppard DN (2009) Gating of the CFTR Cl- channel by ATP-driven nucleotide-binding domain dimerisation. J Physiol 587:2151-2161.

Johnson BA and Blevins RA (1994) NMR View: a computer program for the visualization and analysis of NMR data. J Biomol NMR 4:603-614.

Lewis HA, Buchanan SG, Burley SK, Conners K, Dickey M, Dorwart M, Fowler R, Gao X, Guggino WB, Hendrickson WA, et al. (2004) Structure of nucleotide-binding domain 1 of the cystic fibrosis transmembrane conductance regulator. $E M B O J \mathbf{2 3}$ : $282-293$

Liu F, Zhang Z, Csanady L, Gadsby DC, and Chen J (2017) Molecular structure of the human CFTR ion channel. Cell 169:85-95.e88.

Loo TW, Bartlett MC, and Clarke DM (2010) The V510D suppressor mutation stabilizes DeltaF508-CFTR at the cell surface. Biochemistry 49:6352-6357.

Loo TW, Bartlett MC, and Clarke DM (2013) Corrector VX-809 stabilizes the first transmembrane domain of CFTR. Biochem Pharmacol 86:612-619.

Mendoza JL, Schmidt A, Li Q, Nuvaga E, Barrett T, Bridges RJ, Feranchak AP, Brautigam CA, and Thomas PJ (2012) Requirements for efficient correction of $\triangle$ F508 CFTR revealed by analyses of evolved sequences. Cell 148:164-174.

Mense M, Vergani P, White DM, Altberg G, Nairn AC, and Gadsby DC (2006) In vivo phosphorylation of CFTR promotes formation of a nucleotide-binding domain heterodimer. $E M B O J \mathbf{2 5}: 4728-4739$.

Mornon JP, Hoffmann B, Jonic S, Lehn P, and Callebaut I (2015) Full-open and closed CFTR channels, with lateral tunnels from the cytoplasm and an alternative position of the F508 region, as revealed by molecular dynamics. Cell Mol Life Sci 72:1377-1403.

Mornon JP, Lehn P, and Callebaut I (2009) Molecular models of the open and closed states of the whole human CFTR protein. Cell Mol Life Sci 66:3469-3486.

Newman ME, Strogatz SH, and Watts DJ (2001) Random graphs with arbitrary degree distributions and their applications. Phys Rev E Stat Nonlin Soft Matter Phys 64:026118.

O'Boyle NM, Banck M, James CA, Morley C, Vandermeersch T, and Hutchison GR (2011) Open Babel: an open chemical toolbox. J Cheminform 3:33

Okiyoneda T, Veit G, Dekkers JF, Bagdany M, Soya N, Xu H, Roldan A, Verkman AS, Kurth M, Simon A, et al. (2013) Mechanism-based corrector combination restores $\Delta$ F508-CFTR folding and function. Nat Chem Biol 9:444-454.

Ostedgaard LS, Baldursson O, and Welsh MJ (2001) Regulation of the cystic fibrosis transmembrane conductance regulator $\mathrm{Cl}-$ channel by its $\mathrm{R}$ domain. J Biol Chem 276:7689-7692.

Proctor EA, Kota P, Aleksandrov AA, He L, Riordan JR, and Dokholyan NV (2015) Rational coupled dynamics network manipulation rescues disease-relevant mutant cystic fibrosis transmembrane conductance regulator. Chem Sci (Camb) 6: 1237-1246.

Protasevich I, Yang Z, Wang C, Atwell S, Zhao X, Emtage S, Wetmore D, Hunt JF, and Brouillette CG (2010) Thermal unfolding studies show the disease causing F508del mutation in CFTR thermodynamically destabilizes nucleotide-binding domain 1. Protein Sci 19:1917-1931.

Rabeh WM, Bossard F, Xu H, Okiyoneda T, Bagdany M, Mulvihill CM, Du K, di Bernardo S, Liu Y, Konermann L, et al. (2012) Correction of both NBD1 energetics and domain interface is required to restore $\triangle$ F508 CFTR folding and function. Cell 148:150-163. 
Ren HY, Grove DE, De La Rosa O, Houck SA, Sopha P, Van Goor F, Hoffman BJ, and Cyr DM (2013) VX-809 corrects folding defects in CFTR through action on membrane-spanning domain 1. Mol Biol Cell 24:3016-3024.

Riordan JR, Rommens JM, Kerem B, Alon N, Rozmahel R, Grzelczak Z, Zielenski J, Lok S, Plavsic N, Chou JL, et al. (1989) Identification of the cystic fibrosis gene: cloning and characterization of complementary DNA. Science 245:1066-1073.

Schrodinger L (2010) The PyMOL Molecular Graphics System, version 1.3. Schrödinger, Cambridge, MA.

Selvaratnam R, Chowdhury S, VanSchouwen B, and Melacini G (2011) Mapping allostery through the covariance analysis of NMR chemical shifts. Proc Natl Acad Sci USA 108:6133-6138.

Serohijos AW, Hegedus T, Aleksandrov AA, He L, Cui L, Dokholyan NV, and Riordan JR (2008) Phenylalanine-508 mediates a cytoplasmic-membrane domain contact in the CFTR 3D structure crucial to assembly and channel function. Proc Natl Acad Sci USA 105:3256-3261.

Sinha C, Zhang W, Moon CS, Actis M, Yarlagadda S, Arora K, Woodroofe K, Clancy JP, Lin S, Ziady AG, et al. (2015) Capturing the direct binding of CFTR correctors to CFTR by using click chemistry. Chembiochem 16:2017-2022.

Thibodeau PH, Richardson, 3rd JM, Wang W, Millen L, Watson J, Mendoza JL, Du K, Fischman S, Senderowitz H, Lukacs GL, et al. (2010) The cystic fibrosis-causing mutation deltaF508 affects multiple steps in cystic fibrosis transmembrane conductance regulator biogenesis. J Biol Chem 285:35825-35835.

Trott O and Olson AJ (2010) AutoDock Vina: improving the speed and accuracy of docking with a new scoring function, efficient optimization, and multithreading. $J$ Comput Chem 31:455-461.
Van Goor F, Straley KS, Cao D, González J, Hadida S, Hazlewood A, Joubran J, Knapp T, Makings LR, Miller M, et al. (2006) Rescue of DeltaF508-CFTR trafficking and gating in human cystic fibrosis airway primary cultures by small molecules. Am J Physiol Lung Cell Mol Physiol 290:L1117-L1130.

Van Goor F, Hadida S, Grootenhuis PD, Burton B, Stack JH, Straley KS, Decker CJ, Miller M, McCartney J, Olson ER, et al. (2011) Correction of the F508del-CFTR protein processing defect in vitro by the investigational drug VX-809. Proc Natl Acad Sci USA 108:18843-18848.

Vernon RM, Chong PA, Lin H, Yang Z, Zhou Q, Aleksandrov AA, Dawson JE, Riordan JR, Brouillette CG, Thibodeau PH and Forman-Kay JD (2017) Mutational stabilization of the second nucleotide-binding domain (NBD2) of CFTR yields soluble protein and insight into NBD2 disease-causing mutations. J Biol Chem, under review.

Wang C, Protasevich I, Yang Z, Seehausen D, Skalak T, Zhao X, Atwell S, Spencer Emtage J, Wetmore DR, Brouillette CG, et al. (2010) Integrated biophysical studies implicate partial unfolding of NBD1 of CFTR in the molecular pathogenesis of F508del cystic fibrosis. Protein Sci 19:1932-1947.

Zhang Z and Chen J (2016) Atomic structure of the cystic fibrosis transmembrane conductance regulator. Cell 167:1586-1597.e9.

Address correspondence to: Julie D. Forman-Kay, Room 20.9713, Molecular Medicine, Hospital for Sick Children, 686 Bay Street, Toronto, ON M5G 0A4, Canada. E-mail: forman@sickkids.ca 